\title{
INVESTIGATING 3D RECONSTRUCTION METHODS FOR SMALL ARTIFACTS
}

\author{
V. Evgenikou, A. Georgopoulos \\ National Technical University of Athens, School of Rural and Surveying Engineering, Lab. of Photogrammetry \\ Zografou Campus, Heroon Polytechniou 9, 15780, Zografou, Athens, Greece \\ vevge@hotmail.com,drag@ central.ntua.gr \\ Commission V, WG V/4
}

KEY WORDS: Laser Scanning, SfM, 3Dmodels, Artifacts

\begin{abstract}
:
Small artifacts have always been a real challenge when it comes to 3D modelling. They usually present severe difficulties for their $3 \mathrm{D}$ reconstruction. Lately, the demand for the production of 3D models of small artifacts, especially in the cultural heritage domain, has dramatically increased. As with many cases, there are no specifications and standards for this task. This paper investigates the efficiency of several mainly low cost methods for 3D model production of such small artifacts. Moreover, the material, the color and the surface complexity of these objects id also investigated. Both image based and laser scanning methods have been considered as alternative data acquisition methods. The evaluation has been confined to the 3D meshes, as texture depends on the imaging properties, which are not investigated in this project. The resulting meshes have been compared to each other for their completeness, and accuracy. It is hoped that the outcomes of this investigation will be useful to researchers who are planning to embark into mass production of 3D models of small artifacts.
\end{abstract}

\section{INTRODUCTION}

Modern technological advancements have enabled the production of three dimensional models of small objects and artifacts of cultural heritage value. At the same time the demand for such three dimensional reconstructions is increasing as they are used in a variety of applications in archaeological studies, education, tourism and many more. Today many different techniques are available for completing this task effectively. These techniques range from image based methods to scanning hardware and software using either laser or common light. Their implementation cost also spreads over a wide price range and so do the results as far as accuracy, completeness and reliability are concerned. The success rate of all these methods depends on the equipment used, on the methodology itself and, of course on the object properties. By this its shape complexity, surface color and material are implied. This issue has not been extensively investigated in the past albeit for larger objects (Toschi et al. 2014); hence this paper is trying to add another piece of relevant information to the puzzle of the multitude of methods and of hardware and software capable of producing three dimensional models of artifacts of different shape and nature.

\section{MOTIVATION}

As digital technologies advance rapidly, digital libraries for cultural heritage assets have evolved, e.g. Europeana (www.europeana.eu). However, up to now only 2D material, i.e. images are stored in these repositories. The first ever 3D object uploaded in Europeana is the 3D model of a Byzantine Church in Cyprus (Sofocleous et al. 2006). In the last years all the more museums and cultural heritage repositories of any kind demand the mass $3 \mathrm{D}$ digitization of monuments and artifacts. This is by no means a trivial task, as all these items present a variety of shapes, a multitude of surface colors, a diversity of materials.

In addition, for many years terrestrial laser scanning has shown a rapid progress and its use spread rapidly over many applications including documentation of cultural heritage assets (Gonzo et al., 2007; Koska \& Kremen, 2013; Mandferdini \& Galassi, 2013). However, in the last decade photogrammetric algorithms have been automated with the contribution of the computer vision community and hence image based techniques have gained the lost ground in the competition for point cloud generation. It is believed that perhaps they already have certain advantages over scanning methods as they are more cost effective, sometimes faster, equally efficient and accurate and they are capable of providing texture to the 3D models (Skarlatos \& Kiparissi 2012). Hence, when it comes to mass production of $3 \mathrm{~d}$ models of artifacts, users are facing a dilemma as to which method is most suitable to use.

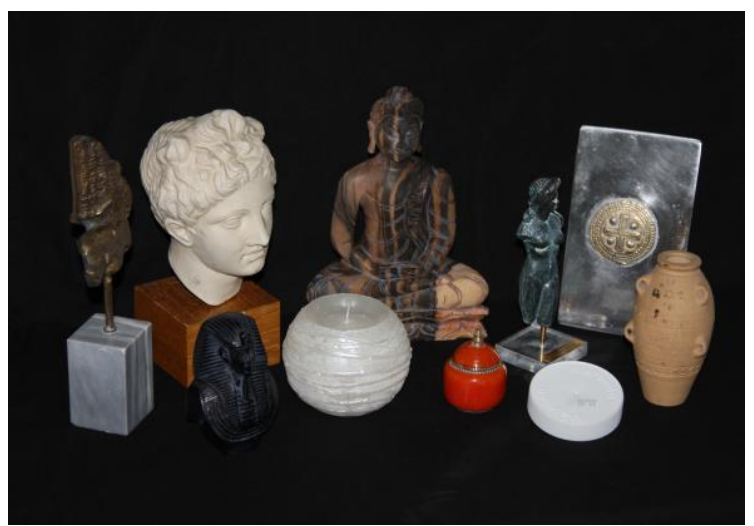

Figure 1: The collection of different artifacts tested (from left to right: Brass, Marble, Plaster, Mahogany, Wax, Wood, Porcelain, Bronze, Plastic, Metal, Clay) 
For that purpose ten different artifacts were carefully selected presenting a multitude of geometry and shape complexity, of texture and a variety of materials (Figure 1). They included small ornamental objects with surfaces made of metal, plastic, wax, wood, marble, plaster and other materials or combination thereof. Some of them had shiny surfaces, a fact which presented additional problems. The main purpose of the project was to investigate the efficiency of the various methods tested to produce the three dimensional surface. Hence image texture was not included in the investigation as it is mainly related to the properties of the digital camera. Except for the marble base in case an object was standing on a base of different material, this was excluded from the investigation.

\section{METHODOLOGY}

\subsection{D data acquisition methods}

Nowadays 3D reconstruction usually implies point cloud generation and processing. Technological advances have enabled the development of a lot of methods for $3 \mathrm{~d}$ data acquisition. The methods available today for producing three dimensional models are many, diverse and mainly could be categorized into two main groups. Passive methods record the electromagnetic energy, i.e. visible light, emitted by the objects to be documented. Active methods, on the other hand, emit electromagnetic energy, e.g. laser beams, infrared light etc., and record its reflection form the object (Remondino \& El-Hakim, 2006; Bianco et al, 2013).

Several of these methods, representative of the wide range have been selected for the test. Laser and structured light scanners, web based services for image based scanning, a commercial software applying Structure-from-Motion (SfM) algorithm and a contemporary total station with scanning capabilities were employed.

Seven different methods were used to generate $3 \mathrm{D}$ point clouds of the artifacts chosen. Those methods belong to two main categories. The first category includes the image based methods (IBM) i.e. passive methods, which are either automated or semiautomated, while the second category includes a variety of active scanners. Specifically:

- Automated image based methods (IBM): 123DCatch software by Autodesk ${ }^{\circledR}$ (now replaced by ReCap), Arc3D web service, MS Photosynth software

- Semi-automated image based methods (IBM): Photoscan software by Agisoft ${ }^{\circledR}$

- Structured Light Scanner: SL2 by XYZRGB ${ }^{\circledR}$ Inc.

- Triangulation based $3 D$ laser scanner: Next Engine ${ }^{\circledR}$

- Time-of-flight $3 D$ laser scanner: Topcon ${ }^{\circledR}$ IS imaging station

All objects were scanned in such a way in order to produce the three dimensional surface in the most efficient way for the comparison to be objective

\subsection{Image Based Methods}

Theoretically, the generation of a point cloud can be achieved faster and cheaper through the image based methods (IBM), while the quality and accuracy of the result depends on image scale and pixel size. Practically, image based methods are proven to be more efficient while facing light-absorbing and dark colored materials, but less efficient while facing items with low textured surfaces (Remondino \& El-Hakim, 2006). Specular and/or very smooth materials have negative effect to the quality of the point cloud, regardless of the method used. In an attempt to overcome those problems, most objects were sprayed with chalk and/or talc powder in order to create texture and to reduce shine and disturbing reflections.

Image acquisition was very carefully planned, in order to ensure best results and objectivity. The object was placed on an elevated stool and two Bowens studio flashes were employed in order to achieve the best lighting conditions (Figure 2). The images were taken with a Canon EOS 1D MIII full frame DSLR with a resolution of $21 \mathrm{Mpixel}$ and a fixed lens of $50 \mathrm{~mm}$ nominal principal distance. Also, the image overlap was 80 $90 \%$, the photographed object appeared focused in the biggest part of each capture, the angle between the shots was less than 10 degrees and the surrounding area remained stable. For ensuring even illumination of the objects a set of two Bowens ${ }^{\mathbb{B}}$ studio flashes was also employed.

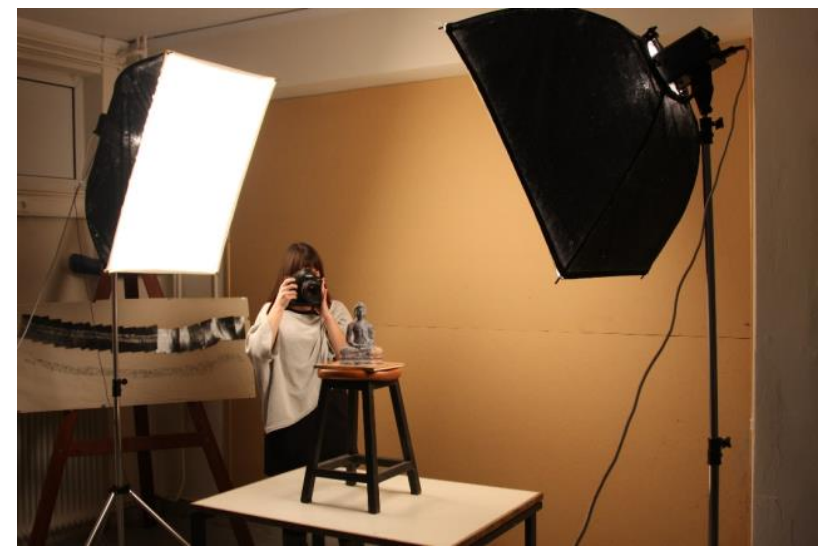

Figure 2: Image acquisition

The number of the captured images for each object varies and depends on the characteristics of each artifact. Blurry images were excluded and the rest were loaded to the software for processing. The results were obtained a few hours later. An example of the procedure described above appears in Figure 3.

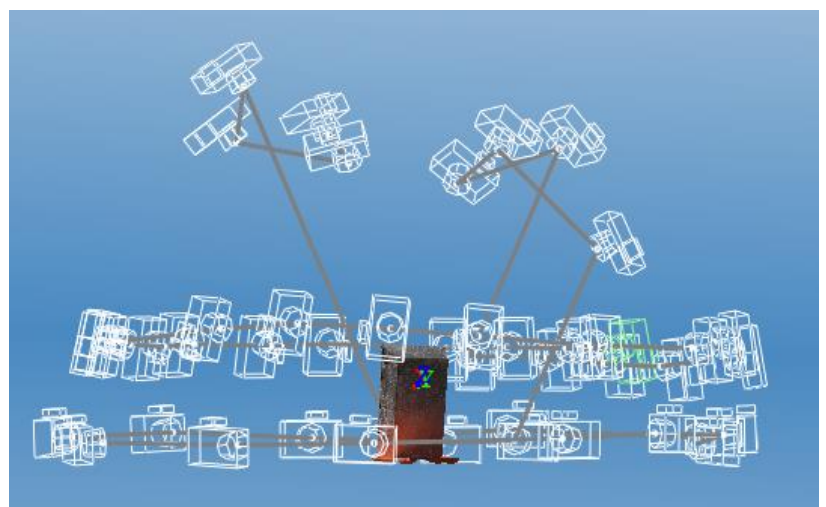

Figure 3: Camera pose determination by 123DCatch

The images acquired for each artifact were uploaded to the 3 web based services, i.e. 123DCatch, Arc3D and Photosynth. The point cloud registration was performed by the service itself, as the user has practically no control. Similarly, in the case of 
Agisoft Photoscan, the images were aligned by the software and the best possible point cloud and dense mesh were produced.

\subsection{Scanning}

As already mentioned three different scanners were used for data acquisition. In each case the system parameters were set in such a way as to achieve the highest possible accuracy and density of the generated clouds.

\subsubsection{SL2 XYZRGB ${ }^{\circledR}$ Structured Light Scanner}

The SL2 structured laser scanner by XYZRGB ${ }^{\circledR}$ (Valanis et al. 2009, Valanis et al. 2010) is composed by a projector and two cameras (DSLR or Machine Vision) and is connected to a computer running the proprietary software (Figure 4). The cameras were placed at the desired positions, i.e. so that a baseto-distance ratio of approximately 1:3 was ensured. The whole system needs to be calibrated for each different setup. Due to the different artifact sizes, two different setups were required and hence two calibrations were performed.

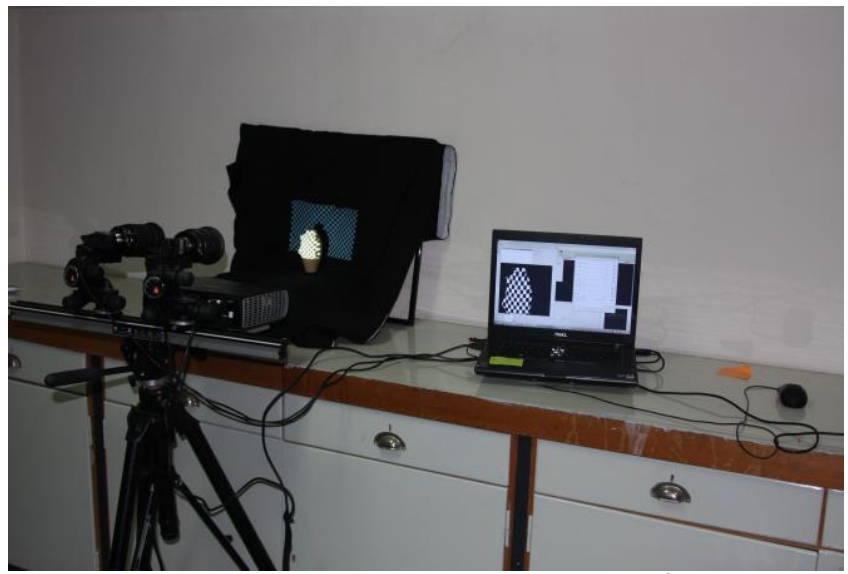

Figure 4: SL2 scanner by XYZRGB ${ }^{\circledR}$

Each artifact was placed in front of a black background, as structured laser scanners in general cannot determine points on black surfaces. The camera parameters, i.e. exposure values for the DSLR's and brightness, contrast for the machine vision cameras etc. were specified. In order to scan the whole surface of each object, it was manually rotated in between the scans. The scanning process for every object lasted 3 hours. Meshes were created from the generated point clouds using the system's software. These meshes were subsequently exported for further processing in Meshlab.

\subsubsection{NEXT ENGINE 3D Laser Scanner}

The Next Engine 3D laser scanner is composed of the main scanner hardware and a connected to the scanner rotating base, on which the objects are placed (Figure 5). For each scan several necessary parameters should be specified, like distance, object brightness, cloud density, positions and number of scans. Each object was scanned in approximately 20 different rotated positions. All the generated meshes were cleaned from unwanted faces and afterwards they were aligned both semi automatically. The merged model was exported to Meshlab for further processing. The scanning, repairing and merging of each model was completed within 5 hours approximately.

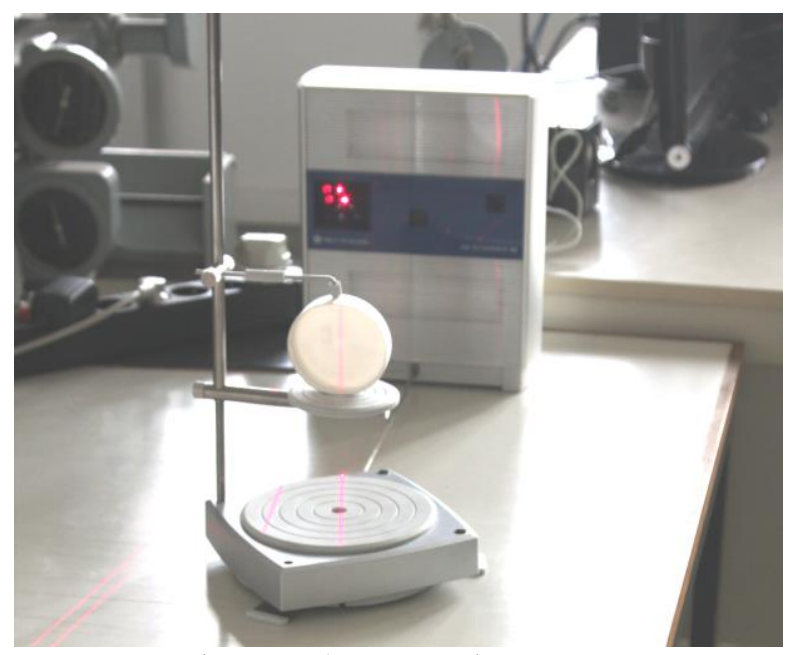

Figure 5: The next Engine scanner

\subsubsection{TOPCON IS Imaging Station}

The Topcon IS total station (http://www.topcon positioning.com/products/total-stations) is one of the first such instruments to incorporate imaging and scanning capabilities. The manufacturer claims distance accuracy $1 \mathrm{~mm} \pm(10 \mathrm{~mm} \pm$ $\pm 10 \mathrm{ppm}), 1.3 \mathrm{MP}$ camera and $20 \mathrm{pts} / \mathrm{sec}$ scanning speed. Considering the imaging station's limited measurement and accuracy capability to obtain a high density $3 \mathrm{D}$ point cloud, this method was used for demonstration purposes and it was decided to scan only a single side of each artifact. The Topcon IS was placed $2.20 \mathrm{~m}$ away from the objects, an arbitrary local coordinate system was created and the scanning parameters were set in order to generate an accurate and dense point cloud to the best of the instrument's capabilities. The data acquisition for each object lasted approximately 40min.

\subsection{Data processing}

\subsubsection{Data preparation}

Depending on the used method, the result was a point cloud (Topcon IS), a group of point clouds (Photosynth), a group of meshes (SL2 XYZRGB, Next Engine) or a merged mesh (123DCatch, Arc3D, Agisoft Photoscan). Topcon IS and Photosynth proved unable to produce a mesh. Hence only point clouds were processed from these two 3D data sources.

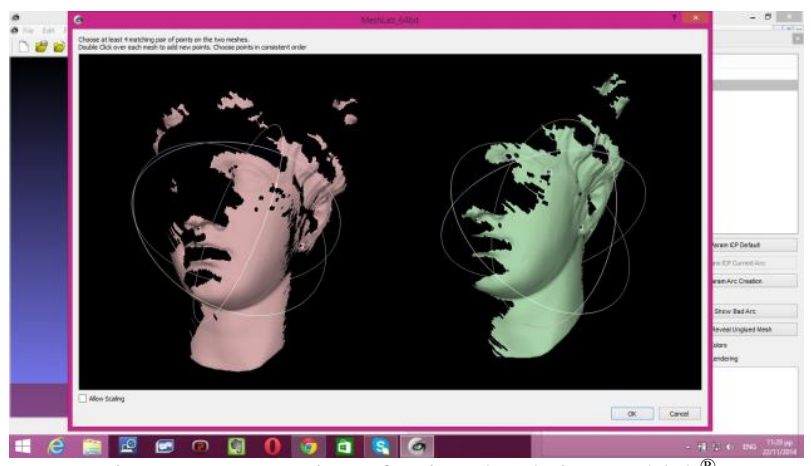

Figure 6: Processing of point clouds in Meshlab ${ }^{\circledR}$

For all other methods meshes were produced and were subsequently processed in Meshlab (Figure 6). This software has been chosen because it is freeware and it offers a large variety of processing options (http://meshlab.sourceforge.net). 
Before that step, all meshes that contained more than 3 million points were subsampled in the Geomagic Studio v.12 software, as Meshlab cannot accommodate point clouds larger than that. This subsampling has been very carefully performed in order not to discard valuable information of the surface.

\subsubsection{Mesh processing}

The following processing stages were performed in Meshlab software:

- Cleaning. The various methods generated points not only from the surface of the artifacts but also from items in the surrounding area. Those points were selected and cropped.

- Alignment. Points were picked and matched manually in order to align the meshes or in order to improve the images' alignment whenever possible. From the Image Based Methods only 123DCatch provided this option. The alignment was optimized automatically.

- Merging. All the partial meshes of each method were merged into a single mesh in order to create a unique model.

- Surface Reconstruction. The points and normals of a cloud were used to build a surface using the Poisson Surface reconstruction approach. The parameters were fixed at the following values as, after experimentation, it was proven that they lead to the most accurate results that Meshlab can produce within a reasonable amount of time.

- Octree Depth: 10,

- Solver Divide: 8 ,

- Samples per Node: 1 ,

- Surface offsetting: 1 .

This approach creates a surface with no holes, so the extra parts ought to be cropped.

- Repairing. The models were repaired by the removal of duplicate and zero area faces duplicated and unreferenced vertices and faces from non-manifold edges.

\section{EVALUATION OF RESULTS}

The resulting three dimensional models were then compared and evaluated for their qualitative and quantitative properties in order to attempt to draw some conclusions relating to the efficiency and accuracy capabilities of each different 3D reconstruction method in conjunction with the material and the geometric complexity of each object. In addition some proposals for the amelioration of the resulting three dimensional models are also attempted.

For the quantitative comparison, highly accurate measurements on the objects were performed with specialized instrumentation. These measurements were also performed on the three dimensional models and the results are presented, discussed and evaluated (Table 1). For comparison the three dimensional models specialized freely available software, i.e. CloudCompare $^{\circledR}$ was used (www.cloudcompare.org). It has been established that MS Photosynth and the Topcon total station are rather poor in performance for producing any of the three dimensional models. On the other hand the structured light scanner, contrary to the initial expectations failed in certain cases to produce complete point clouds. However it was rather successful in many other cases tested. The point clouds produced with the NextEngine ${ }^{\circledR}$ scanner are acceptable. Specific difficulties were presented by highly complex objects. The web services, i.e. Arc3D and 123DCatch, performed satisfactorily with many of the objects, while presenting certain weaknesses in shiny objects and artifacts with no texture. This was also the problem of the commercial software and in certain cases it was overcome by spraying the objects with chalk powder in order to create some kind of texture.

\subsection{Qualitative evaluation}

The qualitative evaluation was performed through the careful observation of the created 3D models. The parameters taken into account were:

- Total or partial point cloud generation

- Surface distortions

- The amount and the size of the existing holes

- Surface roughness

The following conclusions were reached for each method of 3D point cloud acquisition.

\subsubsection{DCatch}

The service failed to create a complete model only in the case of the wooden artifact. All the produced meshes have deformations that appear to be significant on the 3D models of the plastic and the wax item due to low texture quality. On the other hand, the majority of the details were modeled and no holes were located.

\subsubsection{Arc3D}

The images of the plastic and the marble object did not lead to the creation of a model. Also, the created models were full of large holes and had great distortions.

\subsubsection{Photosynth}

The low density of the point clouds that were produced with this method characterizes it as incapable for visualizing the details of the object (Kersten, 2012). The surface construction was possible with post processing, but it was very timeconsuming and required the implementation of many different software and algorithms.

\subsubsection{Agisoft Photoscan}

The Photoscan software created successfully a 3D model for every artifact. The surface roughness appears to be lower than it should. Also, tiny holes and slight deformations are detected all over the models. The alignment of the images of the metal and the wax artifact led to meshes of even lower quality with great deformations. Nevertheless, it was the only method that generated an acceptable model of the candle.

\subsubsection{Next Engine}

The Next Engine scanner creates high-quality meshes with minimum noise. Great deformations are detected only on the 3D model of the wooden object. Also, the point cloud of the brass artifact contained a lot of noise that led to high surface roughness. Additionally, black texture inserts noise to the cloud. The scanning of the candle led to the acquisition of a very lowdense point cloud that basically contained just noise. 


\subsubsection{SLS}

Generally, the method leads to great results. The scanning of objects with too dark color (not entirely black) and/or reflective surfaces, results to the creation of 3D models with plenty of imperfections, such as deformations, large holes and misaligned meshes. The black objects cannot be scanned, so the method failed to acquire a point cloud from the mahogany item. It also failed to generate a model from the candle.

\subsubsection{Topcon IS}

The generated point clouds deviate significantly from the form of the objects regardless of the material and has such low density that it cannot be converted to a mesh to produce any of the $3 \mathrm{D}$ models.

Representative examples of what is described above are the meshes that were acquired from the clay artifact (Figure 7).

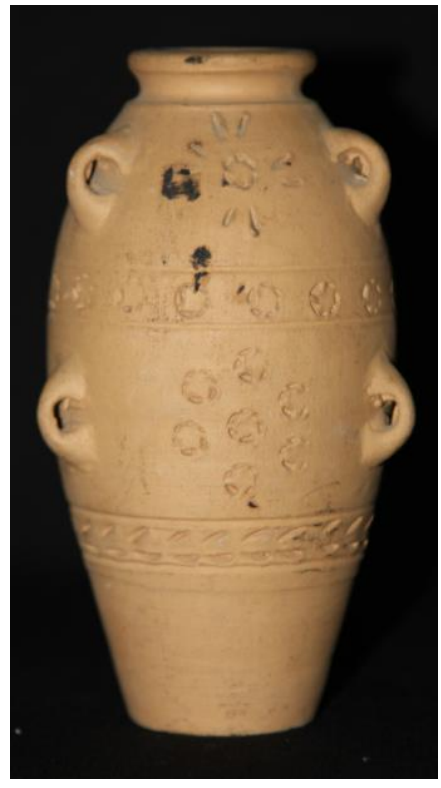

(a)

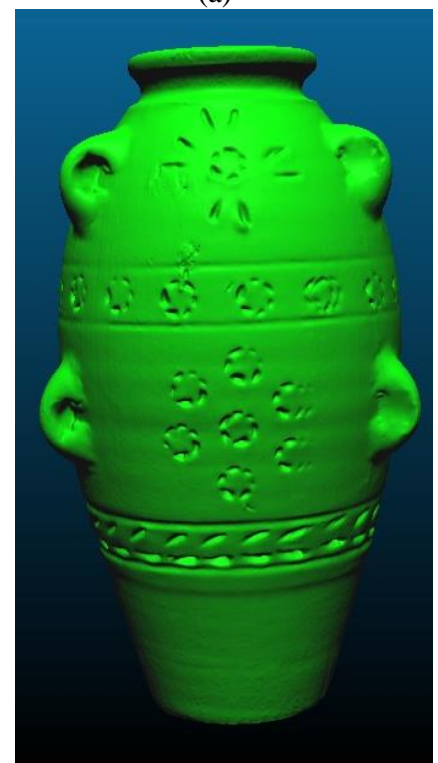

(e)

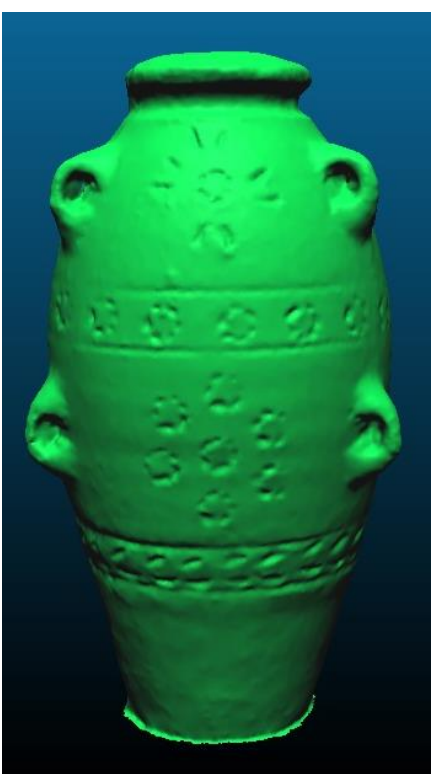

(b)

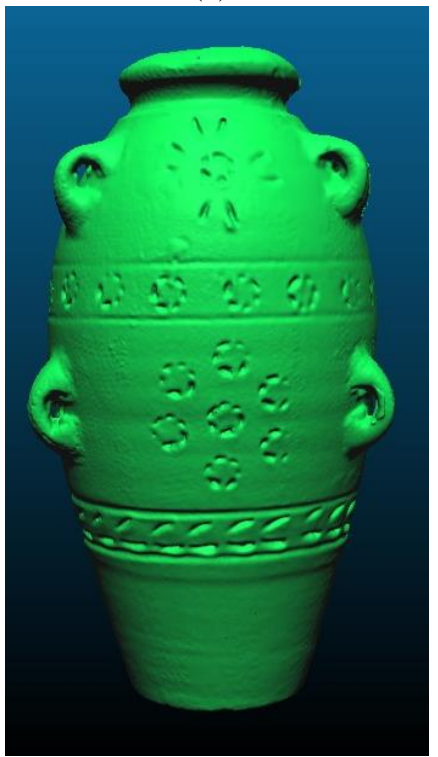

(f)

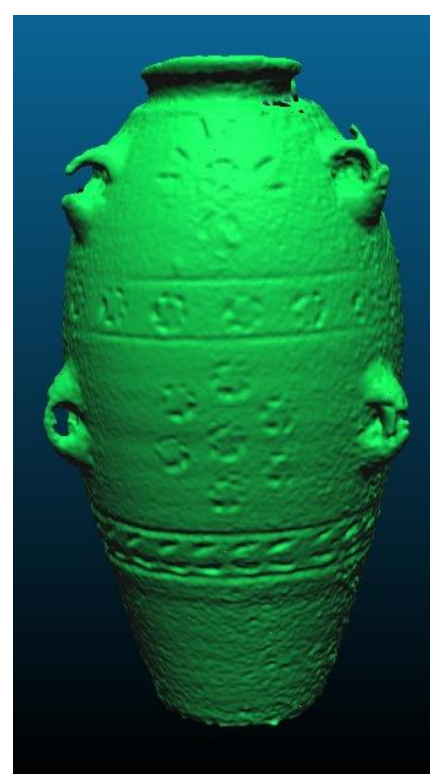

(c)

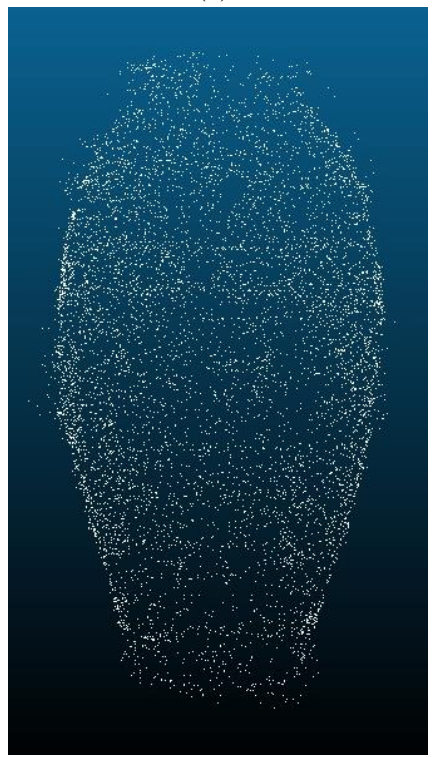

(g)

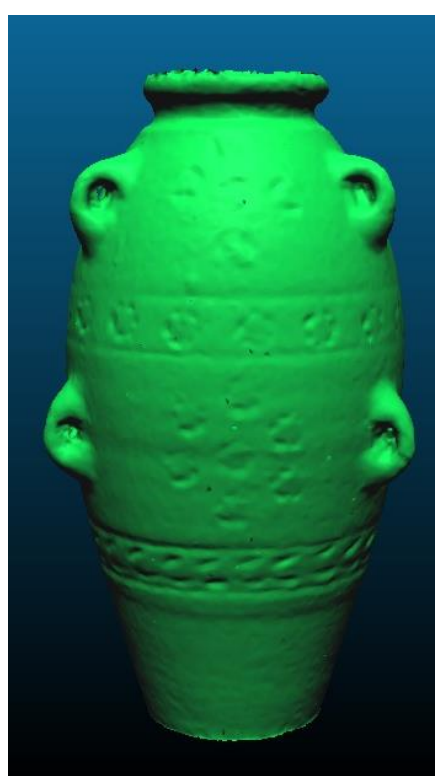

(d)

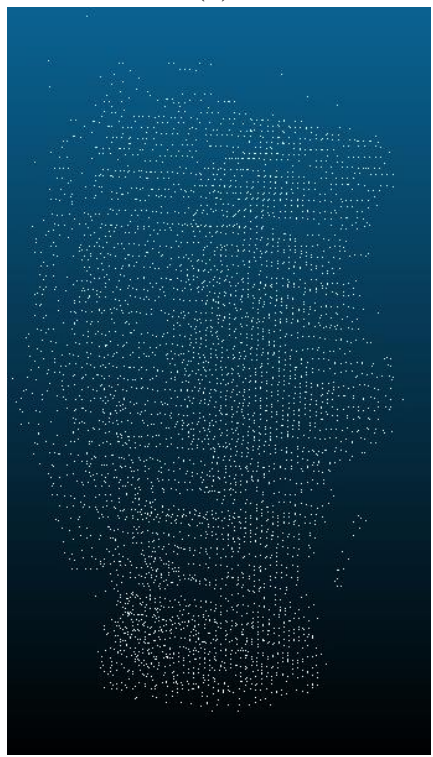

(h)

Figure 7: (a) Clay Object, (b) 3D model created from 123dCatch, (c) 3D model created from Arc3D, (d) 3D model created from Photoscan, (e) 3D model created from Next Engine, (f) 3D model created from SL2, (g) Point cloud generated by Photosynth, (h) Point cloud generated by Topcon IS

\subsection{Quantitative evaluation}

To evaluate the accuracy and the model scale of the created models highly accurate measurements were performed on the artifacts. For that purpose, a digital caliper with accuracy of $0.01 \mathrm{~mm}$ was used to measure approximately fourteen control distances on each object. The respective distances were also digitally measured on three dimensional models. Those distances varied in length and were dispersed on the surfaces of the artifacts. Due to the low density of the point clouds, the distances could not be measured on the clouds generated by Photosynth and Topcon IS. 
The first step was to calculate the scale of each model from equation [1]. Regardless of the fact that scanners generate scaled models, every model's scale $(\mathrm{K})$ was calculated and amended in order to reach more objective conclusions.

$\mathrm{K}=\left(\mathrm{S} 1 / \mathrm{S} 1^{\prime}+\mathrm{S} 2 / \mathrm{S} 2{ }^{\prime}+\ldots \ldots+\mathrm{S}(\mathrm{n}-\mathrm{m}) / \mathrm{S}(\mathrm{n}-\mathrm{m})^{\prime}\right) /(\mathrm{n}-\mathrm{m})$

S: distance measured using the digital caliper S': distance measured within CloudCompare $\mathrm{n}$ : the amount of the control measurements $\mathrm{m}$ : the amount of the distances that cannot be accurately located due to model deformations.

Additionally, the mean error of each model was estimated from equations [2] and [3], and the standard deviation was also calculated. The results are presented in Table $1 \mathrm{in} \mathrm{mm}$. Some values are missing because the method failed to produce a point cloud.

Error: $\mathrm{Ei}=\mathrm{Si}-\mathrm{Si}{ }^{*} * \mathrm{~K}$

Mean model error: $\mathrm{M} . \mathrm{E}=(\mathrm{E} 1+\mathrm{E} 2+\ldots \ldots+\mathrm{En}) / \mathrm{n}$

\begin{tabular}{|c|c|c|c|c|c|c|c|c|c|c|}
\hline & \multicolumn{2}{|c|}{ 123DCatch } & \multicolumn{2}{|c|}{ ARC3D } & \multicolumn{2}{c|}{ PhotoScan } & \multicolumn{2}{c|}{ Next Engine } & \multicolumn{2}{c|}{ SL2 } \\
\hline & Mean & St.D & Mean & St.D & Mean & St.D & Mean & St.D & Mean & St.D \\
\hline PLASTER & 0,17 & 1,21 & $\mathbf{0 , 0 9}$ & $\mathbf{1 , 0 7}$ & 0,02 & 1,60 & 0,14 & 1,34 & 0,39 & 1,19 \\
\hline WOOD & $\mathbf{- 0 , 3 0}$ & $\mathbf{1 , 7 0}$ & $-1,10$ & 2,81 & $-0,36$ & 2,47 & 0,35 & 4,66 & $-1,16$ & 2,38 \\
\hline PORCELAIN & $-0,43$ & 1,85 & $-2,47$ & 4,73 & $-0,36$ & 2,43 & 0,17 & 2,08 & $\mathbf{0 , 0 9}$ & $\mathbf{2 , 0 0}$ \\
\hline CLAY & 0,52 & 0,95 & 0,50 & 1,24 & $-0,58$ & 1,38 & $\mathbf{- 0 , 1 3}$ & $\mathbf{0 , 6 4}$ & $-0,34$ & 0,82 \\
\hline MAHOGANY & $\mathbf{0 , 0 4}$ & $\mathbf{0 , 8 9}$ & $-0,38$ & 0,95 & 0,10 & 1,03 & $-0,17$ & 0,68 & - & - \\
\hline METAL & 0,40 & 0,66 & $-0,20$ & 1,24 & 1,26 & 1,83 & $\mathbf{- 0 , 3 0}$ & $\mathbf{0 , 5 8}$ & 0,63 & 1,36 \\
\hline MARBLE & 0,02 & 1,08 & - & - & $\mathbf{0 , 0 0}$ & $\mathbf{0 , 7 3}$ & $-0,09$ & 0,70 & $-0,16$ & 1,17 \\
\hline BRASS & 0,12 & 0,93 & $-0,15$ & 0,92 & 0,13 & 0,64 & $\mathbf{0 , 1 0}$ & $\mathbf{0 , 9 2}$ & 0,33 & 1,11 \\
\hline BRONZE & $-0,32$ & 1,42 & 0,11 & 1,87 & 0,03 & 1,33 & $-0,19$ & 1,11 & $\mathbf{0 , 0 8}$ & $\mathbf{0 , 4 6}$ \\
\hline PLASTIC & $-0,76$ & 1,18 & - & - & 0,78 & 1,21 & $\mathbf{0 , 3 2}$ & $\mathbf{0 , 5 4}$ & 0,41 & 0,65 \\
\hline
\end{tabular}

Table 1: 3D Models' Mean Error and Standard Deviation (in mm)

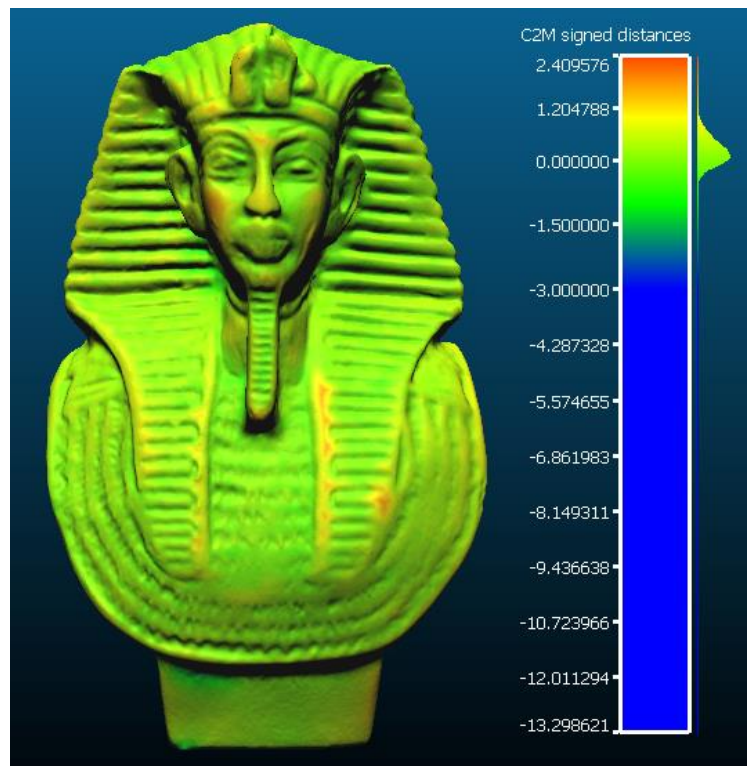

Figure 8: Computing distances between aligned models

After the identification of the models with the greater accuracy in combination with the most favorable standard deviation (highlighted in Table 1), they were compared with all other models the same object that were created using different methods. Within CloudCompare ${ }^{\circledR}$ the $3 \mathrm{D}$ models were scaled, aligned and the distances between the aligned models were computed in $\mathrm{mm}$ (Figure 8 ).
Control distances were could not be measured either on the candle or on the clouds acquired from it, because of the almost complete lack of distinguishable features. However, from the qualitative evaluation of the models, it was clear that the model generated by Photoscan was the only acceptable, so it was used as base for the quantitative comparisons. The scales of the created models were adjusted automatically.

Scale adjustments were also performed for the Photosynth's and Topcon's point clouds, although, in the case of clouds acquired with the Topcon IS, it was rarely successful. Hence the relevant values are not shown in Table 2 . These adjustments justify the low values shown on table 2 .

The values computed by the Cloud Compare (Table 2) were slightly different from the expected. Those divergences are justified due to the following reasons:

- The characteristics of each artifact set limitations on the choice of the control measurements which could have led to the incorrect estimation of the scale factors and the mean errors.

- The surface distortions and holes definitely affected the results

- The boundaries of the objects were not identical. 


\begin{tabular}{|c|c|c|c|c|c|c|c|c|c|c|c|c|c|c|}
\hline & \multicolumn{2}{|c|}{ 123DCatch } & \multicolumn{2}{|c|}{ Arc3D } & \multicolumn{2}{|c|}{ PhotoScan } & \multicolumn{2}{|c|}{ Photosynth } & \multicolumn{2}{|c|}{ Topcon IS } & \multicolumn{2}{|c|}{$\begin{array}{c}\text { Next } \\
\text { Engine } \\
\end{array}$} & \multicolumn{2}{|c|}{ SLS } \\
\hline & MD & St.D. & MD & St.D. & MD & St.D. & MD. & St.D. & MD & St.D. & MD & St.D & MD & St.D \\
\hline PLASTER & $-0,10$ & 0,54 & $\mathrm{~B}$ & B & 0,12 & 0,79 & 0,10 & 0,68 & $-0,17$ & 3,25 & 0,09 & 0,61 & $-1,31$ & 0,64 \\
\hline WOOD & $\mathrm{B}$ & B & 0,10 & 0,92 & $-0,07$ & 1,54 & $-0,01$ & 1,15 & $-0,04$ & 3,86 & 0,24 & 1,89 & 0,09 & 0,89 \\
\hline PORCELAIN & 0,70 & 0,35 & 3,00 & 1,41 & 0,27 & 0,27 & 0,00 & 0,68 & - & - & 0,40 & 0,24 & B & $\mathrm{B}$ \\
\hline CLAY & 1,96 & 0,74 & 1,19 & 0,32 & $-0,63$ & 0,48 & 0,04 & 0,95 & - & - & B & B & 0,36 & 0,32 \\
\hline MAHOGANY & B & B & 0,26 & 0,47 & $-0,16$ & 0,45 & $-0,07$ & 1,17 & - & - & 0,18 & 0,43 & - & - \\
\hline METAL & 0,43 & 0,26 & $-0,32$ & 0,57 & 0,99 & 1,00 & 0,03 & 0,46 & - & - & $\mathrm{B}$ & $\mathrm{B}$ & 0,63 & 0,60 \\
\hline MARBLE & 0,07 & 0,12 & - & - & B & B & $-0,07$ & 0,56 & - & - & $-0,11$ & 0,53 & 0,15 & 0,54 \\
\hline BRASS & 0,04 & 0,24 & $-0,12$ & 0,39 & 0,25 & 0,29 & 0,06 & 0,57 & - & - & B & B & 1,12 & 0,59 \\
\hline BRONZE & $-0,16$ & 0,47 & 0,94 & 2,34 & $-0,01$ & 0,40 & 0,10 & 0,82 & - & - & $-0,18$ & 0,42 & B & B \\
\hline PLASTIC & $-0,02$ & 0,38 & - & - & 0,02 & 0,30 & 0,00 & 0,74 & - & - & $\mathrm{B}$ & $\mathrm{B}$ & 0,00 & 0,15 \\
\hline WAX & 0,01 & 1,08 & $-0,03$ & 3,04 & B & B & $-0,38$ & 1,92 & $-0,69$ & 5,08 & - & - & - & - \\
\hline
\end{tabular}

\section{CONCLUDING REMARKS}

As expected, there is no clear advantage for any method investigated for being suitable for small artifact 3D modelling given the variety of materials included in this investigation. Moreover, the list of parameters taken into account in this project was by no means complete. It has been endeavoured to include in this research the materials which appear more often in such cases. In addition the methods used are not all low cost, but they constitute a representative sample of methods that are available today for this very important task.
Geometric problems due to the complex surface of the objects causing occlusions are apparent in almost all artifacts with such properties. However, this problem may easily be resolved using Image based methods, as one can easily take suitable pictures to image points in the occluded area.

In Table 3 the characteristics of the methods used are presented giving some measure of general evaluation. Acquisition and processing time are approximate estimates, as they varied a little from object to object. User friendliness is based on subjective criteria. It could differ if other users were involved.

\begin{tabular}{|c|c|c|c|c|c|c|c|}
\hline & 123DCatch & Arc3D & Photosynth & Photoscan & Topcon IS (one side) & Next Engine & SLS \\
\hline Cost of equipment (\$) & - & - & - & 3500 & 30000 & 3000 & 80000 \\
\hline Acquisition time (h) & 1.5 & 1.5 & 1.5 & 1.5 & 1 & 2 & 3,5 \\
\hline Processing time (h) & 4 & 6 & 1 & 6 & 0.5 & 6 & 8 \\
\hline User-friendliness & $4 / 5$ & $5 / 5$ & $5 / 5$ & $4 / 5$ & $6 / 5$ & 6500000 & 4000000 \\
\hline $\begin{array}{c}\text { Average cloud density } \\
\text { (before decimation) }\end{array}$ & 100000 & 180000 & 13500 & 160000 & 6500 & \\
\hline \multicolumn{7}{|c|}{ Table 3: Characteristics of the methods used } \\
\hline
\end{tabular}

From the analysis presented above, which was based on the qualitative and quantitative evaluation, an overall ranking was attempted. In Table 4 the relevant marks are presented. These marks are based on both the objective and the subjective assessment.

\begin{tabular}{|c|c|c|c|c|c|c|c|c|}
\hline & 123DCatch & ARC3D & Photosynth & Photoscan & TOPCON IS & $\begin{array}{c}\text { Next } \\
\text { Engine }\end{array}$ & SLS & MEAN \\
\hline PLASTER & 8,5 & 7,5 & 3 & 9,5 & 1 & $\mathbf{1 0}$ & 8,5 & $\mathbf{6 , 9}$ \\
\hline WOOD & 5 & 5 & 2 & $\mathbf{8 , 5}$ & 1 & 5,5 & 6 & $\mathbf{4 , 7}$ \\
\hline PORCELAIN & 6,5 & 0 & 2 & 6 & 0 & $\mathbf{8 , 5}$ & 7,5 & $\mathbf{4 , 4}$ \\
\hline CLAY & 6 & 3,5 & 1,5 & 6,5 & 0,5 & 8 & $\mathbf{8 , 5}$ & $\mathbf{4 , 9}$ \\
\hline MAHOGANY & 6,5 & 5,5 & 2 & 7 & 0,5 & $\mathbf{9 , 5}$ & - & $\mathbf{5 , 2}$ \\
\hline METAL & 7,5 & 5 & 2 & 5,5 & 1 & $\mathbf{8}$ & 5,5 & $\mathbf{4 , 9}$ \\
\hline MARBLE & 8,5 & - & 2,5 & 9 & 0 & $\mathbf{9 , 5}$ & 7 & $\mathbf{6 , 1}$ \\
\hline BRASS & 8,5 & 8 & 2 & 8 & 0,5 & $\mathbf{1 0}$ & 5 & $\mathbf{6}$ \\
\hline BRONZE & 6 & 2 & 1 & 8 & 0 & $\mathbf{8 , 5}$ & 7,5 & $\mathbf{4 , 7}$ \\
\hline PLASTIC & 3,5 & - & 0 & 7,5 & 0 & $\mathbf{8}$ & 7,5 & $\mathbf{4 , 4}$ \\
\hline WAX & 5,5 & 3 & 1 & 7 & 0,5 & - & - & $\mathbf{3 , 4}$ \\
\hline MEAN & $\mathbf{6 , 5}$ & $\mathbf{4 , 4}$ & $\mathbf{1 , 7}$ & $\mathbf{7 , 5}$ & $\mathbf{0 , 5}$ & $\mathbf{8 , 6}$ & $\mathbf{7}$ & \\
\hline
\end{tabular}

Table 4: Overall assessment of the methods used 
This table depicts the best method for each different material and summarizes in the last row the overall evaluation of each method for the different artifacts. In the last column of Table 4, the behavior of each different material is shown as a mean of the assessment of all methods used, or all methods that produced an acceptable result. This, of course, is just an indication of the mean reaction of the specific material to all scanning methods.

The conclusions of this project could assist a potential user for the selection of the most suitable methodology for the three dimensional reconstruction of small artifacts taking into account the special properties of the objects, such as their complex geometry and shape and their material and color properties. In addition the cost of equipment and related instrumentation should also be taken into account. Moreover the cost and time necessary for data acquisition and processing are also a decisive factor.

\section{REFERENCES}

\subsubsection{References from Books:}

\subsubsection{References from Other Literature:}

Bianco, G., Gallo, A., Bruno, F., Muzzupappa, M., 2013, A Comparative Analysis between Active and Passive Techniques for Underwater 3D Reconstruction of Close-Range Objects, Sensors 2013, 13, 11007-11031; doi:10.3390/s130811007

Gonzo, L., Voltolini, F., Girardi, S., Rizzi, A., Remondino, F., El-Hakim, S.F., 2007, Multiple Techniques Approach to the 3D Virtual Reconstruction of Cultural Heritage, Eurographics Italian Chapter Conference 2007.

Kersten, Th., Lindstaedt, M., 2012: Automatic 3D Object Reconstruction from Multiple Images for Architectural, Cultural Heritage and Archaeological Applications Using Open-Source Software and Web Services. Photogrammetrie, Fernerkundung, Geoinformation (PFG) 2012 (6).

Koska, B., Kremen, T., 2013. The combination of laser scanning and structure from motion technology for creation of accurate exterior and interior Orthophotos of St. Nicholas baroque church. International Archives of the Photogrammetry, Remote Sensing and Spatial Information Sciences, Volume XL-5/W1, 2013. 3D-ARCH 2013 - 3D Virtual Reconstruction and Visualization of Complex Architectures, 25 - 26 February 2013, Trento, Italy

Manferdini, A., Galassi, M., 2013, Assessments for 3D Reconstructions of Cultural Heritage using Digital Technologies, International Archives of the Photogrammetry, Remote Sensing and Spatial Information Sciences, Volume XL5/W1, 3D-ARCH 2013 - 3D Virtual Reconstruction and Visualization of Complex Architectures, Trento, Italy.

Remondino, F., EL-Hakim, S., 2006, Image-Based 3D Modelling: A Review, Swiss Federal Institute of Technology (ETH), Zurich, National Research Council, Ottawa, Canada, The Photogrammetric Record 21(115): 269-291, September 2006.
Skarlatos, D., Kiparissi, S., 2012, Comparison of Laser Scanning, Photogrammetry and SFM-MVS Pipeline Applied in Structures and Artificial Surfaces, Dept. of Civil Engineering and Geomatics, Cyprus University of Technology, ISPRS Annals of the Photogrammetry, Remote Sensing and Spatial Information Sciences, Volume I-3, 2012, XXII ISPRS Congress, Melbourne, Australia

Sofocleous, E., Georgopoulos, A., Ioannides, M., Ioannidis Ch., 2006. "The Geometric Documentation of the Asinou Church in Cyprus", VAST 2006, Lefkosia Cyprus, Oct-Nov. 2006, in: The e-volution of Information Communication Technology in Cultural Heritage (eds. M. Ioannides et al.), p. 138-144 ISBN10: 9638046759 .

Toschi, I., Capra, A., De Luca, L., Beraldin, J.-A., Cournoyer, L., 2014. On the evaluation of photogrammetric methods for dense 3D surface reconstruction in a metrological context. ISPRS Annals of the Photogrammetry, Remote Sensing and Spatial Information Sciences, Volume II-5, 2014 ISPRS Technical Commission V Symposium, 23 - 25 June 2014, Riva del Garda, Italy.

Valanis, A., Georgopoulos, A., Sofronidou M., Hadzilacos Th., 2009. "Scanning for Microns" Proceedings XXII CIPA Symposium, October 11-15, 2009, Kyoto, Japan.

Valanis, A., Georgopoulos, A., Ioannidis, Ch., 2010. "Assessing The Performance of a Structured Light Scanner", International Symposium of ISPRS Comm. V, Newcastle 21-25 June 2010.

\subsubsection{References from websites:}

https://www.nextengine.com (last visited on January 2015) http://www.topconpositioning.com (last visited on January 2015)

http://meshlab.sourceforge.net (last visited on January 2015) www.cloudcompare.org (last visited on January 2015) http://www.123dapp.com/catch (last visited on January 2015) http://www.arc3d.be (last visited on January 2015) https://photosynth.net (last visited on January 2015) http://www.xyzrgb.com (last visited on January 2015) http://www.geomagic.com (last visited on January 2015) http://www.agisoft.com (last visited on January 2015) 